\title{
Parametric Modeling in Food Package Defect Imaging
}

\author{
Qi Tian, Member, IEEE, Bao-Shen Sun, Ayhan Ozguler, Scott A. Morris, \\ and William D. O'Brien, Jr., Fellow, IEEE
}

\begin{abstract}
A novel approach in food package defect detection is proposed based on system identification theory, in which the channel defect detection problem can be regarded as the conventional system identification problem, i.e., estimation of the system impulse response based on the input-output sequence using parametric and nonparametric models. The well-known parametric model ARX has been investigated in this paper. The data are collected with a focused ultrasound transducer $(17.3 \mathrm{MHz}, 6.35-\mathrm{mm}$ diameter, f/2, $173 \mu \mathrm{m}-6 \mathrm{~dB}$ pulse-echo lateral beam width at the focus) scanned over a rectangular grid, keeping the packages in the focus. Performance is measured in terms of detection rate, image contrast, and contrast-to-noise ratio. The results using the ARX model are compared with previous image formation techniques and also compared with the non-parametric method, i.e., spectral analysis. The results show that the ARX model has the comparable detection rate as RFCS and higher detection rate than BAI and RFS (except 6- $\mu \mathrm{m}$ air-filled channel in plastic trilaminate film) for channel in plastic trilaminate film. The ARX model has achieved the moderate contrast enhancement and ranks second in contrast-to-noise ratio enhancement among the compared techniques. The ARX model has a low detection rate for channel defects in aluminum trilaminate film, which shows that its performance is material-dependent. Finally, the parametric method, ARX model demonstrates better performance than the non-parametric method, spectral analysis for food package defect detection.
\end{abstract}

\section{INTRODUCTION}

$\mathrm{C}$ HANNEL leaks and weak seals are two major defects found in the seal region of flexible food packages [1]. Channel leaks might cause a pathway for microbial penetration that eventually results in the spoilage of the product. The weak seal that is generally caused by wrinkles or product involvement in the seal area causes the seal strength to decrease and gives rise to product deterioration during storage [2]. Because both types of defects bring about health hazards and economic loss, it is imperative that packaging be checked for the presence of such defects.

Manuscript received August 4, 1999; accepted December 8, 1999. This work was supported in part by the China Scholarship Council (BSS); the Value-Added Research Opportunities Program, Agricultural Experiment Station, University of Illinois; and the University of Illinois Research Board.

Q. Tian and W. D. O'Brien, Jr. are with Bioacoustics Research Laboratory, Department of Electrical and Computer Engineering, University of Illinois, Urbana, IL 61801 (e-mail: wdo@uiuc.edu).

B. Sun is with the Institute of Acoustics, Chinese Academy of Sciences, Beijing, China.

A. Ozguler and S. A. Morris are with the Packaging Program, Department of Food Science and Human Nutrition, University of Illinois, Urbana, IL 61801.
Previous investigations of food packages have led to several imaging techniques to detect channels in package seals. The backscattered amplitude integral (BAI) imaging technique [3] displays the integral of the envelope-detected RF signal at each transducer position. Channels with diameters of $38 \mu \mathrm{m}$ or larger can be detected reliably using this technique at a center frequency of $17.3 \mathrm{MHz}$. The RF sample (RFS) imaging technique [4], [5] displays, for each transducer position, a single time-gated acoustic pressure value of the received $\mathrm{RF}$ waveform. The selection of only a single time gate requires a priori knowledge of the packaging material, which can be obtained off-line. By selecting only one time point on the received $\mathrm{RF}$ waveform, there is risk that a channel is present but not detected. This risk can be reduced by forming multiple images with several time gates. The RF correlation (RFC) imaging technique [5], [6] requires less specific a priori information and offers potentially higher detectability at a cost of greater computational complexity. The RFC image is created by displaying the normalized correlation coefficients for each transducer position. Depending on the range over which the correlation window is placed, the RFC image has two different versions: one that contains the entire range of the $\mathrm{RF}$ signal (denoted RFCE) and one that contains only a short, specified duration of the RF signal (denoted RFCS). For channels smaller than $15 \mu \mathrm{m}$, the appropriate choice of the correlation window plays an important role in the RFCS technique. Both RFS and RFCS have improved detection rates relative to BAI for channels of $15 \mu \mathrm{m}$ or smaller [5]. However, none of these techniques provide $100 \%$ detection of channel defect smaller than $38 \mu \mathrm{m}$ in diameter. Therefore, additional image formation approaches must be evaluated to determine whether improved detection rates are possible for smaller channels.

In this paper, a novel approach is proposed for food package defect detection based on system identification theory [7], [8]. The system identification problem is to estimate a model of a system from the observed input-output data. There are two kinds of identification methods: parametric methods and non-parametric methods. Parametric identification methods are techniques to estimate parameters in given model structures. Basically, it is a matter of finding (by numerical search) those numerical values of the parameters that give the best agreement between the model's output and that measured. Most common models are difference equation descriptions, such as ARX and ARMAX models [7], [8], as well as all types of linear statespace models. Non-parametric identification methods are 


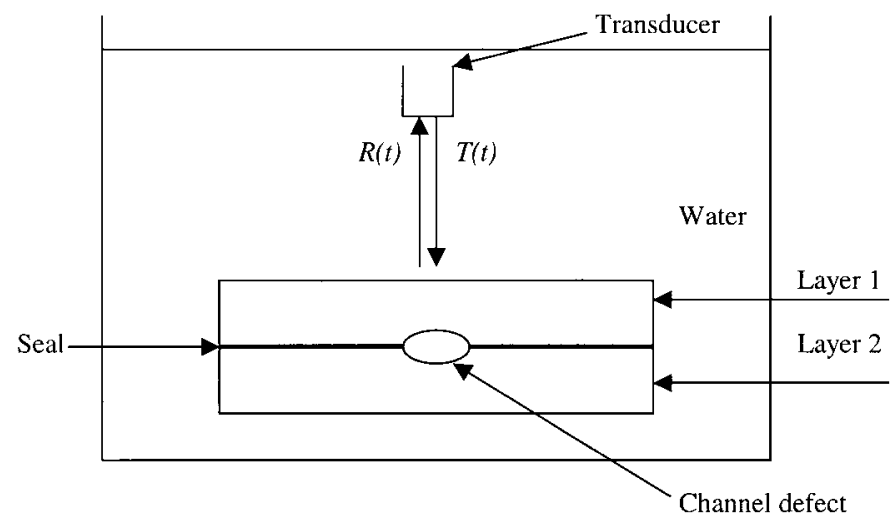

Fig. 1. The system model.

techniques to estimate model behavior without necessarily using a given parametrized model set. Typical nonparametric methods include correlation analysis, which estimates a system's impulse response, and spectral analysis, which estimates a system's frequency response.

The rest of the paper is organized as follows. System model is presented in Section II. In Section III, the general system identification theory is discussed. In Section IV, the image formation of the proposed approach is described. In Section V, the results of using the ARX model are compared with the previous imaging techniques and are also compared with a non-parametric method. Finally, conclusions and suggestions for future work are given in Section VI.

\section{System Model}

The sample preparation and data acquisition are discussed extensively in [3] and [5]. The sample (Fig. 1) is located in a water tank $\left(\sim 20^{\circ} \mathrm{C}\right)$ so that the defect orientation is approximately normal to the sound beam direction. A three-dimensional data set is collected using a nominal 20-MHz focused transducer (V317; Panametrics, Waltham, MA) operating at a center frequency of 17.3 $\mathrm{MHz}(6.35-\mathrm{mm}$ diameter, $\mathrm{f} / 2,173 \mu \mathrm{m}-6 \mathrm{~dB}$ pulseecho lateral beamwidth at the focus). The transducer is shock-excited by a pulser-receiver (Model 5800; Panametrics) with a $300 \mathrm{~V}$ monocycle pulse. The transducer is scanned in two directions collecting pulse-echo RF waveforms spaced $30 \mu \mathrm{m}$ apart in the seal direction and $100 \mu \mathrm{m}$ apart in the transverse direction. The sample is kept in the focal region of the transducer throughout the scan. The signal is captured by digitizing oscilloscope (9354TM; LeCroy, Chestnut Ridge, NY) with a sampling rate of $500 \mathrm{MHz}$, keeping 512 samples for each transducer position. This data set is also used in [4]-[6] to form the BAI, RFS, and RFCS images.

In Fig. 1, assume the transmitted acoustic signal to be $T(t)$ in the time domain and the sample under evaluation be a system of three layers in water [3], [5]. The echo signal $R(t)$ can be written as the convolution of the transmitted acoustic signal $T(t)$ and the impulse response of the data collection system. When the seal region without the defect is scanned, the echo signal $R_{p}(t)$ can be written as

$$
R_{p}(t)=T(t) * h_{w}(t) * h_{1}(t) * h_{s}(t) * h_{2}(t)
$$

where $*$ denotes convolution and $h_{w}(t), h_{1}(t), h_{s}(t)$, and $h_{2}(t)$ are the impulse responses of the water layer between the transducer and sample, the sample layer 1, seal layer (very thin), and sample layer 2, respectively. The reflections from the boundaries between the water layer and the sample are included in the impulse response functions of $h_{1}(t)$ and $h_{2}(t)$.

It is assumed that the impulse response of the perfect seal is an all-pass filter. Then, it can be removed from (1):

$$
R_{p}(t)=T(t) * h_{w}(t) * h_{1}(t) * h_{2}(t) .
$$

As the ultrasonic focal spot moves to the channel defect, the echo signal $R_{d}(t)$ can be written as

$$
R_{d}(t)=T(t) * h_{w}(t) * h_{1}(t) * h_{d}(t) * h_{2}(t)
$$

where $h_{d}(t)$ is the impulse response of the channel defect. Combining (2) and (3) yields

$$
R_{d}(t)=R_{p}(t) * h_{d}(t) .
$$

From (4), the received echo signal that passes through the channel defect is the convolution of the received echo signal, which does not pass through the channel defect, and the impulse response of the channel defect. Because both $R_{d}(t)$ and $R_{p}(t)$ are available from pulse-echo measurements, it is possible to find the impulse response of the channel defect by the process of deconvolution. Now, if we reconsider $R_{d}(t)$ to be the output and $R_{p}(t)$ to be the input of a new system, then $h_{d}(t)$ would be the impulse response of this new system. Therefore, the problem to find the impulse response $h_{d}(t)$ of the system becomes a conventional system identification problem, i.e., estimation of the system impulse response based on the known input-output sequence.

\section{System Identification Theory}

This section discusses the basic system identification theory. For more details about the algorithms and theories of identification, refer to [7], [8]. For more details about systems and signals, refer to [9].

\section{A. Polynomial Representation of Model Structure}

The basic input-output configuration is shown in Fig. 2 . Assuming unit sampling interval, there is an input signal $u(t)$ and output signal $y(t), t=1,2, \ldots, N$. Assuming the signals are related by a linear system, the input-output relationship can be written

$$
y(t)=G(q) u(t)+v(t)
$$




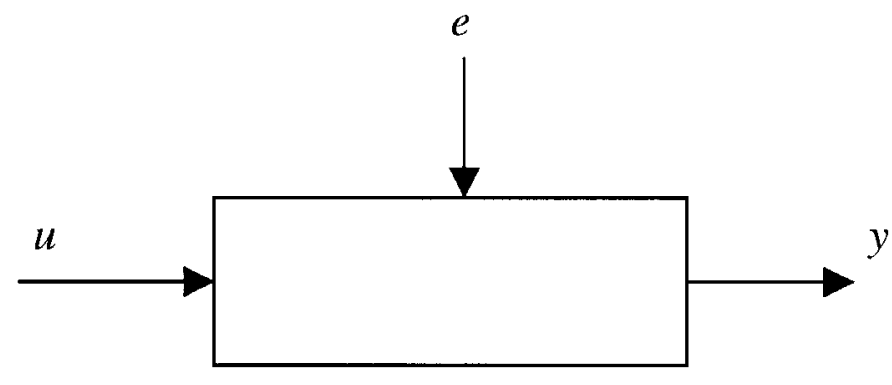

Fig. 2. The input-output configuration.

where $q$ is the shift operator and $G(q)$ is the transfer function of the system. $v(t)$ is the disturbance of the system which can be described as filtered white noise:

$$
v(t)=H(q) e(t)
$$

where $e(t)$ is white noise with variance $\lambda$ and $H(q)$ is the transfer function of the filter. Eq. (5) and (6) together give a time-domain description of the system:

$$
y(t)=G(q) u(t)+H(q) e(t) .
$$

A commonly used parametric model is the ARX model that corresponds to

$$
G(q)=q^{-n k} \frac{B(q)}{A(q)} ; \quad H(q)=\frac{1}{A(q)}
$$

where $B$ and $A$ are polynomials in the delay operator $q^{-1}$ :

$$
\begin{aligned}
& A(q)=1+a_{1} q^{-1}+\ldots \ldots+a_{n a} q^{-n a} \\
& B(q)=b_{1}+b_{2} q^{-1}+\ldots \ldots+b_{n b} q^{-n b+1} .
\end{aligned}
$$

Here, the numbers $n a$ and $n b$ are the orders of their respective polynomials. The number $n k$ is the number of delays from input to output. The model is usually written

$$
A(q) y(t)=B(q) u(t-n k)+e(t)
$$

or explicitly

$$
\begin{aligned}
y(t)+ & a_{1} y(t-1)+\ldots \ldots+a_{n a} y(t-n a)= \\
& b_{1} u(t-n k)+b_{2} u(t-n k-1)+\ldots . . \\
& b_{n b} u(t-n k-n b+1)+e(t) .
\end{aligned}
$$

Note that (11) and (12) apply also to the multivariable case, where $A(q)$ and the coefficients $a_{i}$ become $n y$ by $n y$ matrices, $B(q)$ and the coefficients $b_{i}$ become $n y$ by $n u$ matrices. Here, ny and $n u$ are the number of outputs and inputs, respectively.

Another very common and more general model is the ARMAX structure

$$
A(q) y(t)=B(q) u(t-n k)+C(q) e(t) .
$$

Here, $A(q)$ and $B(q)$ are as in (9) and (10), respectively, and

$$
C(q)=1+c_{1} q^{-1}+\ldots \ldots+c_{n c} q^{-n c} .
$$

An output-error (OE) structure is obtained as

$$
y(t)=\frac{B(q)}{F(q)} u(t-n k)+e(t)
$$

with

$$
F(q)=1+f_{1} q^{-1}+\ldots \ldots f_{n f} q^{-n f} .
$$

The so-called Box-Jenkins (BJ) model structure is given by

$$
y(t)=\frac{B(q)}{F(q)} u(t-n k)+\frac{C(q)}{D(q)} e(t)
$$

with

$$
D(q)=1+d_{1} q^{-1}+\ldots \ldots+d_{n d} q^{-n d} .
$$

All of these models are special cases of the general parametric model structure:

$$
A(q) y(t)=\frac{B(q)}{F(q)} u(t-n k)+\frac{C(q)}{D(q)} e(t) .
$$

Within the structure of (19), virtually all of the usual linear black-box model structures are obtained as special cases. The ARX structure is obtained for $n c=n d=n f=$ 0 . The ARMAX structure corresponds to $n f=n d=0$. The ARARX structure (or the "generalized least squares model") is obtained for $n c=n f=0$; the ARARMAX structure (or "extended matrix model") corresponds to $n f=0$. The OE model is obtained with $n a=n c=n d=0$; the BJ model corresponds to $n a=0$. (See Section 4.2 in [7] for a detailed discussion.)

\section{B. Estimating Parametric Models}

Given a description (7) and having observed the inputoutput data $u, y$, the (prediction) errors $e(t)$ in (7) can be computed as

$$
e(t)=H^{-1}(q)[y(t)-G(q) u(t)] .
$$

These errors are, for given data $y$ and $u$, functions of $G$ and $H$. These in turn are parametrized by the polynomials in (11)-(19).

The most common parametric identification method is to determine estimates of $G$ and $H$ by minimizing

$$
V_{N}(G, H)=\sum_{t=1}^{N} e^{2}(t)
$$

that is

$$
\left[\hat{\mathrm{G}}_{N}, \hat{\mathrm{H}}_{N}\right]=\arg \min \sum_{t=1}^{N} e^{2}(t) .
$$

This is called a prediction error method. For Gaussian disturbances, it coincides with the maximum likelihood method [7, ch. 7]. 

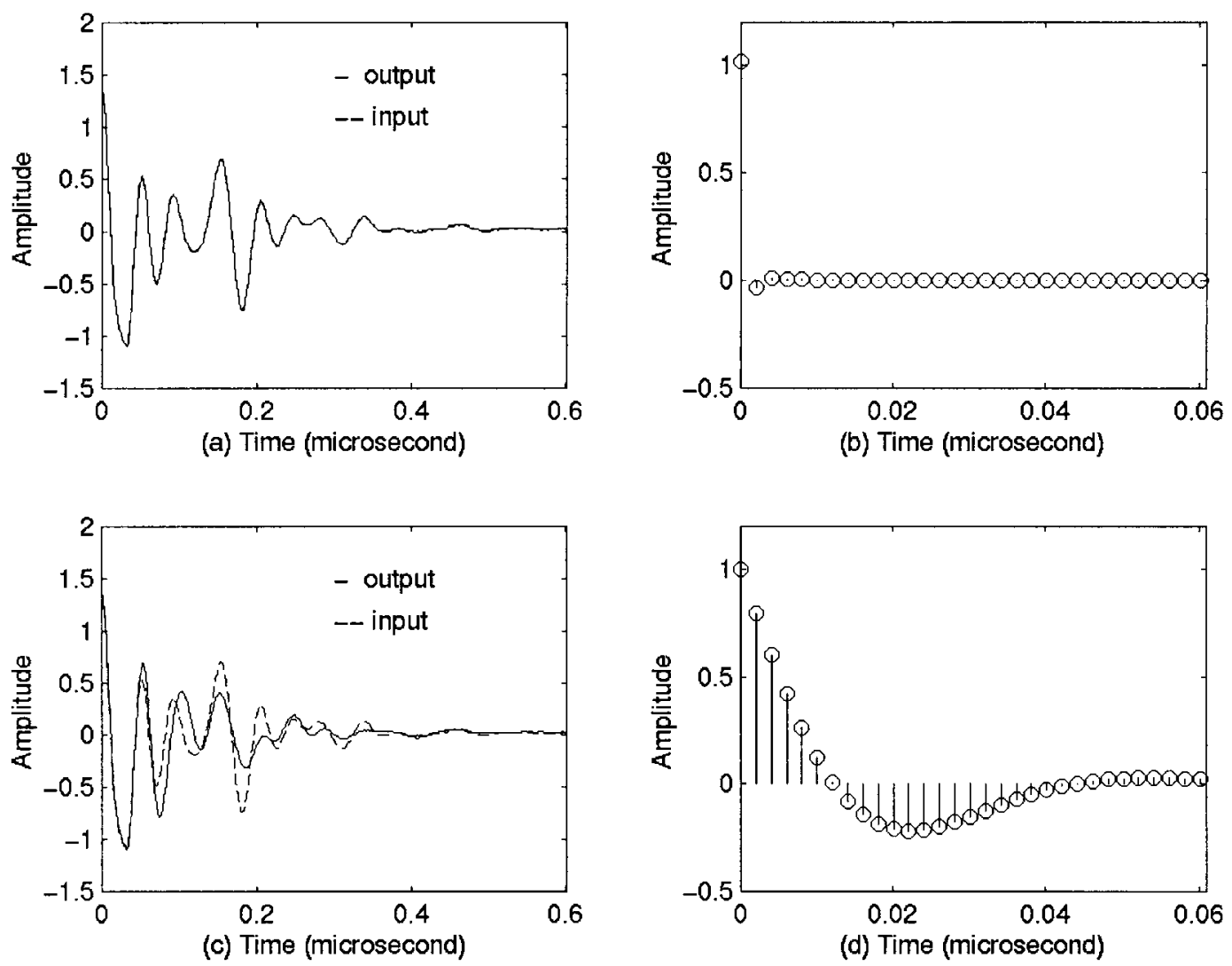

Fig. 3. An example using the ARX model. a) Input and output without channel defect and b) its impulse response. c) Input and output with a $38-\mu \mathrm{m}$, air-filled channel in plastic trilaminate film and d) its impulse response.

\section{IMAGe Formation}

If we consider $R_{p}(t)$, echo from a perfect seal as input to a new system to be identified, and $R_{d}(t)$, echo from a channel defect as output of this new system, then the system impulse response would correspond to the impulse response of the channel defect. To be more general, we model the received RF signal that may or may not pass through the channel defect as an output and the RF signal that does not pass through the channel defect as the input of a new system. It is our assumption that the RF signals that do not pass through the channel defect are similar. Therefore, if a channel defect does not exist, the estimated system impulse response will be a $\delta$ function. Once the parametric model is established, the frequency response of the system to be identified can be calculated from $G$, and its impulse response can be obtained from the inverse Fourier transform of the system frequency response.

In this paper, the ARX model is applied to the food package defect detection problem, and its performance is compared with the previous imaging techniques, i.e., BAI, RFS, and RFCS, and also compared with a non-parametric method, i.e., spectral analysis. For convenience, the image formed using the ARX model is denoted as an ARX image. The image formed using spectral analysis is denoted as an SA image.

To create ARX images, the waveforms at each transducer position are first justified in time to correct for flaws on the surface of the package. This justification is accomplished by aligning the signals at the time instant when the pressure reaches its maximum. This alignment was also done for the RFS and RFCS images [4]-[6]. Second, a reference RF signal is chosen to represent the RF signal not passing through the channel defect, i.e., $R_{p}(t)$ [5], [6].

The input-output of the system without channel defect is plotted in Fig. 3(a). Fig. 3(b) shows the corresponding estimated system impulse response using the ARX model with parameters $n a=2, n b=2$, delay $=0$. Without the channel defect, the output and input are similar, corresponding to a $\delta$ function impulse response in the time domain. Fig. 3(c) shows the input-output of the system with a $38-\mu \mathrm{m}$, air-filled channel in plastic trilaminate film. The corresponding estimated system impulse response is shown in Fig. 3(d). It is obvious that the estimated impulse response is different from a $\delta$ function when a channel defect is present. Thus, the area under the impulse response curve is calculated and used to form the ARX image. The following is the procedure:

- take the absolute value of the impulse response,

- normalize the obtained impulse response, and

- calculate the integral of the normalized impulse response, which is the area under the impulse response curve.

For a $\delta$ function impulse response, i.e., no channel defect, the integral is 1 . The integral is greater than 1 when a 


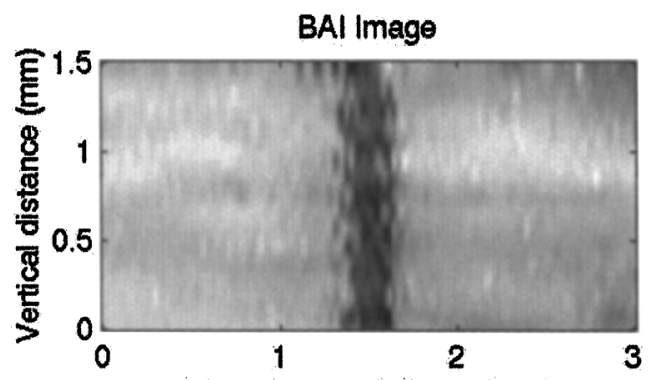

(a) Horizontal distance (mm) RFCS Image

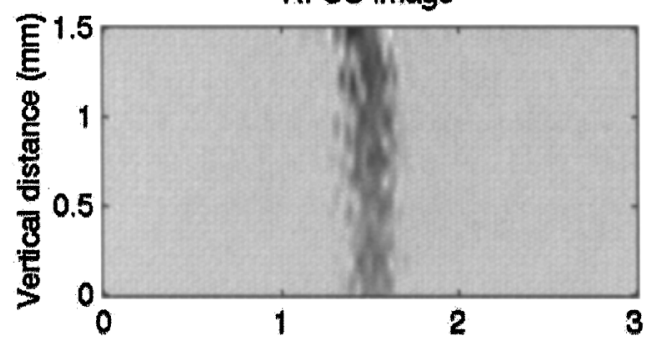

(c) Horizontal distance (mm) ARX Imege

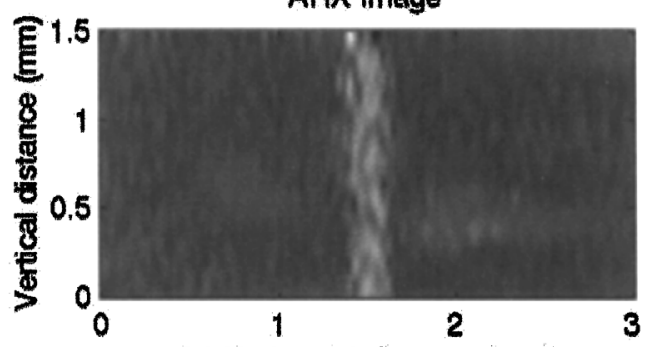

(e) Horizontal distance (mm)

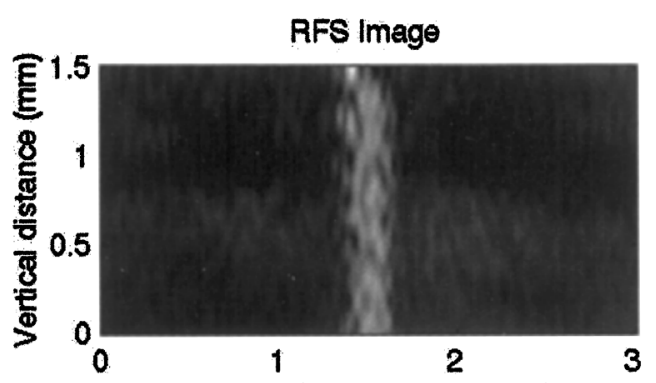

(b) Horizontal distance ( $\mathrm{mm}$ ) SA Image

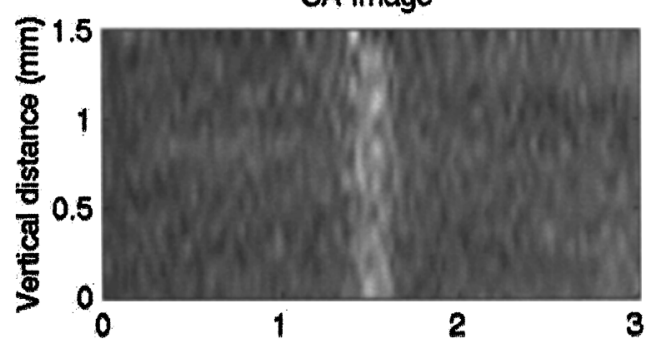

(d) Horizontal distence $(\mathrm{mm})$

Fig. 4. Processed images for 38- $\mu \mathrm{m}$, air-filled channel in plastic trilaminate film: a) BAI image b) RFS Image, $0.164 \mu \mathrm{s}$; c) RFCS image, $t_{1}=0.08$ and $t_{2}=0.12$, where $t_{1}$ and $t_{2}$ are given in terms of microseconds after the pressure maximum; d) SA image; and e) ARX image, $n a=2, n b=2$, delay $=0$.

channel defect exists. The larger the integral, the more likely a channel defect exists. Therefore, the ARX image is created by displaying this integral value for each transducer position forming a 2-D matrix.

The SA image is formed following the same principle as the ARX image, except the impulse response is estimated via spectral analysis instead of ARX model [7].

All calculations are done in MATLAB ${ }^{\circledR}$ on a Sun workstation.

\section{Results}

The data sets used to form the BAI, RFS, and RFCS images in previous work [4]-[6] are also used in this paper to form the ARX and SA images. The same performance measures of detection rate, image contrast, and contrastto-noise ratio (CNR) used in [4]-[6] are also used herein.

Fig. 4 and 5 show examples of two different defects. In Fig. 4, the defect is a $38-\mu \mathrm{m}$ air-filled channel in plastic trilaminate film. The defect is visible in all images. In Fig. 5, the defect is a $6-\mu \mathrm{m}$, water-filled channel in plastic trilaminate film. The defect is visible in the RFS, RFCS, and ARX images and is not detected in the BAI and SA im- ages. These examples show that the ARX model can detect small channels that are not detected by BAI and SA techniques.

Parameter choices for the ARX model affect the image. Fig. 6 shows examples of the ARX images with different parameters. In Fig. 6(a, c, and e), the ARX images are shown for the $38-\mu \mathrm{m}$, water-filled channel in plastic trilaminate film with parameters $n a=2, n b=2$, delay $=0 ; n a=6, n b=6$, delay $=0 ;$ and $n a=10$, $n b=20$, delay $=10$, respectively. The defect is most visible in Fig. 6(a). The defect is not detected in Fig. 6(c), and it is slightly visible in Fig. 6(e). Therefore, lower orders of parameters work well for $38-\mu \mathrm{m}$ defect from our experiments. Fig. 6(b, d, and f) shows the ARX images for $6-\mu \mathrm{m}$, water-filled channel in plastic trilaminate film with parameters $n a=2, n b=2$, delay $=0 ; n a=6$, $n b=6$, delay $=0 ;$ and $n a=10, n b=20$, delay $=10$, respectively. The $6-\mu \mathrm{m}$ defect is not visible with lower orders of parameters [Fig. 6(b and d)] and is detectable with higher orders of parameters [Fig. 6(f)]. Therefore, in this case, higher orders of parameters work well for $6-\mu \mathrm{m}$ defect from our experiments.

We have not done any theoretical investigation for parameter optimization. However, from our limited experi- 

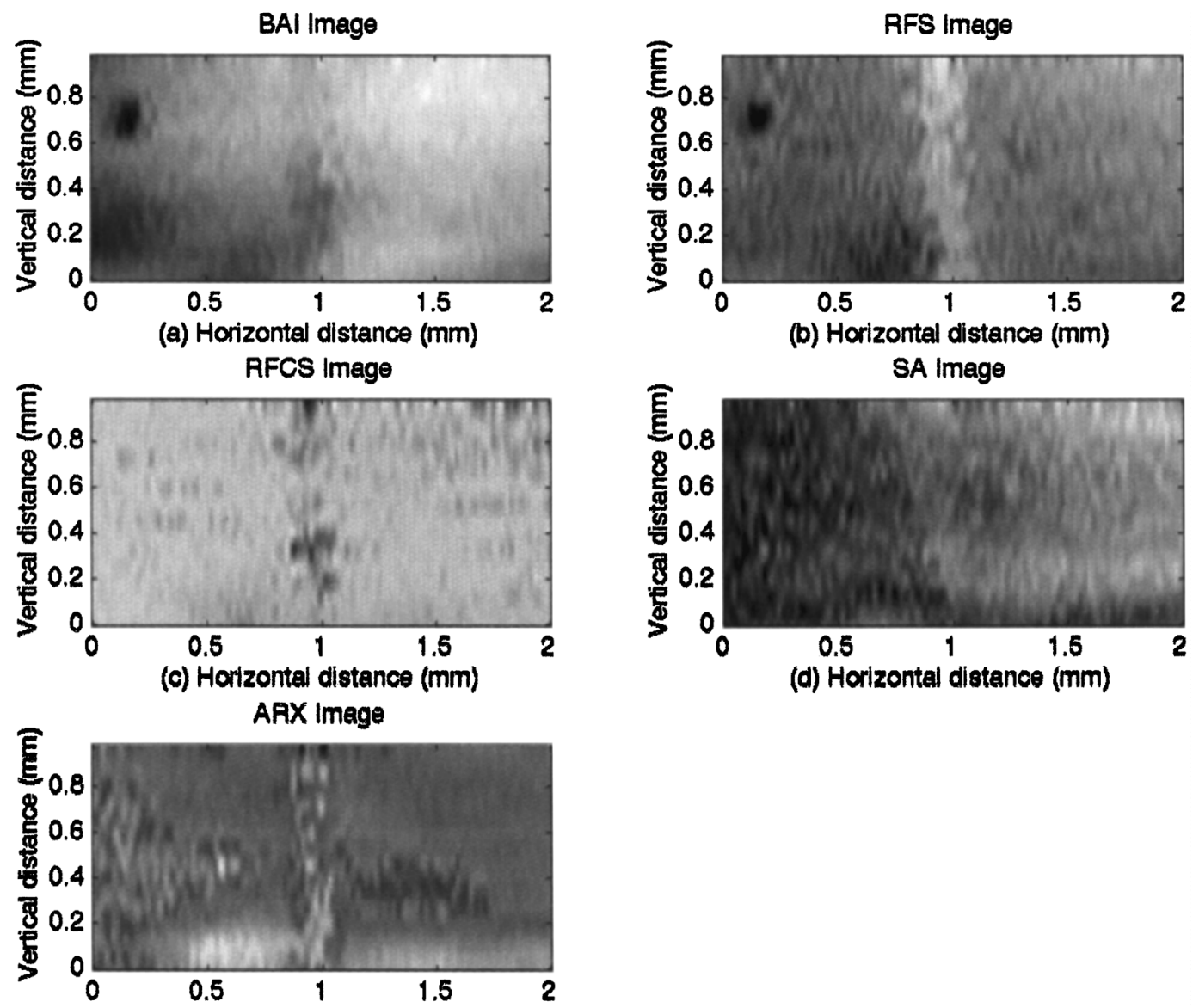

(e) Horizontal distance (mm)

Fig. 5. Processed images for $6-\mu \mathrm{m}$, water-filled channel in plastic trilaminate film: a) BAI image; b) RFS image, $0.082 \mu \mathrm{s}$; c) RFCS image, $t_{1}=0.08$ and $t_{2}=0.12$, where $t_{1}$ and $t_{2}$ are given in terms of microseconds after the pressure maximum; d) SA image; and e) ARX image, $n a=10, n b=20$, delay $=10$.

ence, the lower orders of parameters are more effective for large channels, i.e., $38 \mu \mathrm{m}$ or larger, and higher orders of parameters are more effective for smaller channels, i.e., $15 \mu \mathrm{m}$ or smaller. It is also our experience that with the increasing of parameter orders, the corresponding delay should be increased accordingly.

Table I shows the detection rates for the BAI, RFS, RFCS, SA, and ARX techniques. The detection rate for the BAI, RFS, and RFCS techniques are obtained from [5] and are listed in Table I for comparison purpose. The following conclusions can be drawn. 1) All five techniques reveal $100 \%$ of $50-\mu \mathrm{m}$ channels, regardless of the type of channel defect. 2) The ARX model works well for the plastic trilaminate film wherein the detection rate for the ARX model is comparable with the detection rate for RFCS. The ARX technique is slightly more likely than RFCS to miss a $10-\mu \mathrm{m}$ channel, and slightly less likely than RFCS to miss a $6-\mu \mathrm{m}$ channel. The ARX technique achieves better detection rate than RFS for $10-$ and $15-\mu \mathrm{m}$, air- or water-filled channels in the plastic trilaminate film and has only one less detection than RFS for $6-\mu \mathrm{m}$ air-filled channel in plastic trilaminate film. Finally, the ARX technique achieves better detection rate than BAI and SA techniques for small channels, i.e., $15 \mu \mathrm{m}$ or smaller. 3)
The ARX model has a very low detection rate for the aluminum trilaminate film for most channel sizes except 50and 10- $\mu \mathrm{m}$ air-filled channel in aluminum trilaminate film, which suggests that the performance of the ARX model is material-dependent, a property that may be useful to classify the unknown sample material because all of the other techniques do not appear to be affected by material properties. 4) The non-parametric modeling, i.e., spectral analysis, has a very low or zero detection rate for channels of size $15 \mu \mathrm{m}$ or smaller, regardless of the type of the channel. It also has a very low detection rate for $38-\mu \mathrm{m}$ channels in aluminum trilaminate film.

The contrast versus channel size and CNR versus channel size for air-filled channels in plastic trilaminate film are shown in Fig. 7(a and c), respectively. Because of the fact that spectral analysis has a very low detection rate for small channels, only the BAI, RFS, RFCS, and ARX techniques are compared here. From Fig. 7(a), ARX has moderate contrast enhancement among four techniques. Contrast is a dimensionless quantity in the range between 0 and 1. For large channels, i.e., 38 and $50 \mu \mathrm{m}$, all four techniques have contrasts larger than 0.25 , a contrast large enough to reveal the channel. From Fig. 7(c), RFCS has achieved the largest CNR values among four techniques for 


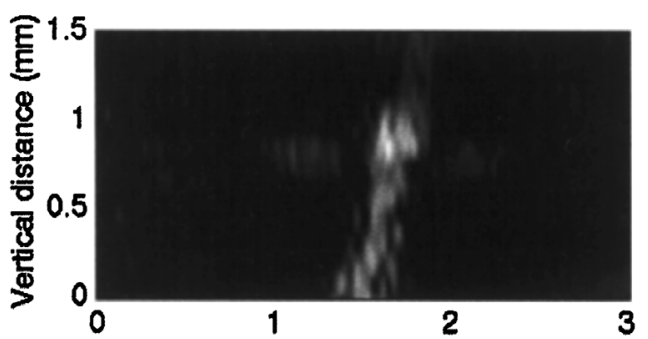

(a) Horizontal distance (mm)

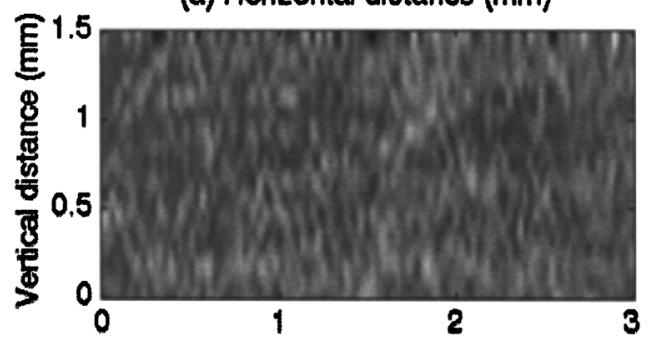

(c) Horizontal distance (mm)

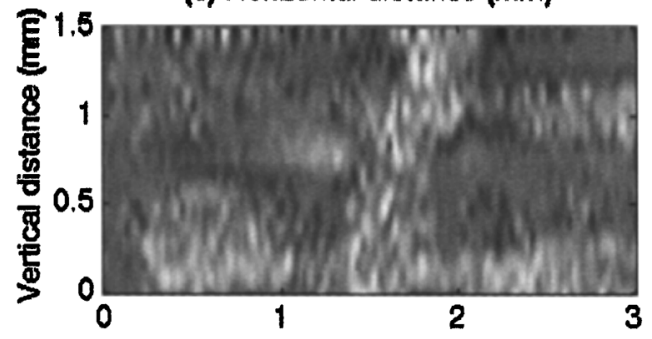

(e) Horizontal distance (mm)

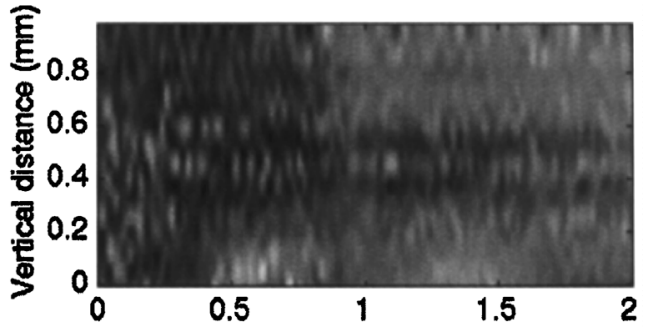

(b) Horizontal distance $(\mathrm{mm})$

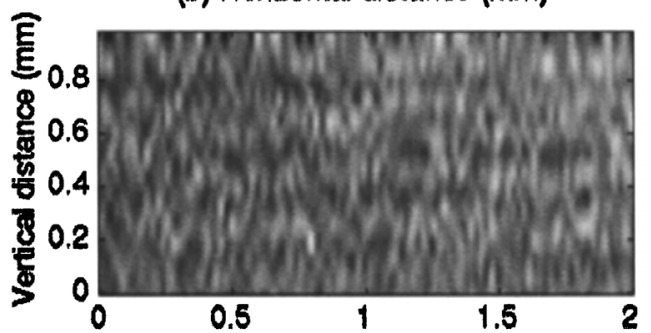

(d) Horizontal distance (mm)

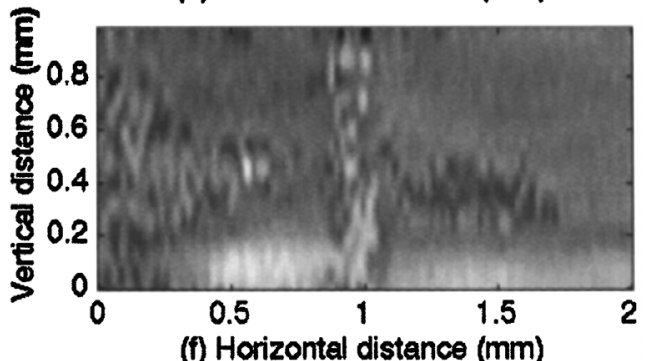

Fig. 6. ARX images: a) $n a=2, n b=2$, delay $=0$; c) $n a=6, n b=6$, delay $=0$; and e) $n a=10$, nb $=20$, delay $=10$ for a 38- $\mu$ m water-filled channel in plastic trilaminate film. b) $n a=2, n b=2$, delay $=0$; d) $n a=6, n b=6$, delay $=0$; and $\mathrm{f}$ ) $n a=10$, $n b=20$, delay $=10$ for a $6-\mu \mathrm{m}$ water-filled channel in plastic trilaminate film.

all channel sizes except $6-\mu \mathrm{m}$. It also has the biggest CNR increment as channel size increases. This fact again demonstrates that RFCS has better capability to smooth the background than the other techniques [5], [6]. This is the advantage of RFCS techniques over other techniques. Because CNR, which includes the effect of speckle and noise, is a more complete description of detectability [10]-[12]. ARX ranks second in terms of CNR value and increment, which also demonstrates good performance to smooth the background.

The contrast versus channel size and CNR versus channel size for water-filled channels in plastic trilaminate film are shown in Fig. 7(b and d), respectively. Clearly, ARX has comparative contrast enhancement with RFCS and has a little higher contrast than RFS, except for $6-\mu \mathrm{m}$ channels. BAI has the lowest contrast enhancement for all channel sizes except $10 \mu \mathrm{m}$. Similarly, as in Fig. 7(c), RFCS and ARX have sharper CNR curves than BAI and RFS as the channel size increases. BAI has a flat CNR curve that shows its least ability to smooth the background among four techniques.

\section{Discussions And Conclusions}

Parametric modeling is applied to detect defects in food package seals. The well-known parametric method, the ARX model, is utilized to estimate the system impulse response that corresponds to the impulse response of the possible channel defect. The applicability of the ARX model for detecting channel defects is demonstrated for plastic trilaminate film with 6-, 10-, 15-, 38-, and 50$\mu \mathrm{m}$ channels filled with air or water. The ARX model is compared with the previous imaging techniques such as BAI, RFS, and RFCS. The results show that, although the ARX model has only the moderate contrast enhancement, the ARX model has achieved the comparable performance as RFCS and performs more effective than BAI and RFS (except $6-\mu \mathrm{m}$ air-filled channels in plastic trilaminate film) in terms of detection rate and CNR enhancement. The performance of the ARX model also shows materialdependent property. It has a very low detection rate for channels of $38 \mu \mathrm{m}$ or smaller, except the $10-\mu \mathrm{m}$ air-filled channel in aluminum trilaminate film. Further investigation of the ARX model on other materials is needed to see whether this material-dependent property could be used to classify unknown sample materials. Finally, when compared with the non-parametric method, the parametric method, i.e., ARX model, performs better than spectral analysis.

It should be noted that parameter optimization has not been done in this work. In general, low orders of parameters work well for large channels, i.e., $38 \mu \mathrm{m}$ or larger, 
TABLE I

Evaluation of Robustness Based on the Detection Rate. The Parameters For the ARX Images ARE $n a=2, n b=2$, delay $=0$ FOR The LARGE Channels, I.E., $38 \mu \mathrm{M}$ OR LARGER; $n a=10, n b=20$, delay $=10$ For the Small Channels, i.E., $15 \mu \mathrm{m}$ or Smaller. The Detection Rate for the BAi, RFS, AND RFCS TeChniques are Obtained from [5] AND ARE Listed in TABle I for Comparison Purposes.

\begin{tabular}{|c|c|c|c|c|c|c|}
\hline $\begin{array}{l}\text { Film/ } \\
\text { channel }\end{array}$ & $\begin{array}{l}\text { Size } \\
(\mu \mathrm{m})\end{array}$ & BAI & RFS & RFCS & $\mathrm{SA}$ & ARX \\
\hline \multirow{5}{*}{$\begin{array}{l}\text { Plastic/ } \\
\text { water }\end{array}$} & 50 & $5 / 5$ & $5 / 5$ & $5 / 5$ & $5 / 5$ & $5 / 5$ \\
\hline & 38 & $5 / 5$ & $5 / 5$ & $5 / 5$ & $4 / 5$ & $5 / 5$ \\
\hline & 15 & $2 / 7$ & $3 / 7$ & $5 / 7$ & $0 / 7$ & $5 / 7$ \\
\hline & 10 & $4 / 5$ & $3 / 5$ & $4 / 5$ & $1 / 5$ & $4 / 5$ \\
\hline & 6 & $1 / 4$ & $3 / 4$ & $2 / 4$ & $0 / 4$ & $3 / 4$ \\
\hline \multirow{5}{*}{$\begin{array}{l}\text { Plastic/ } \\
\text { air }\end{array}$} & 50 & $5 / 5$ & $5 / 5$ & $5 / 5$ & $5 / 5$ & $5 / 5$ \\
\hline & 38 & $5 / 5$ & $5 / 5$ & $5 / 5$ & $5 / 5$ & $5 / 5$ \\
\hline & 15 & $6 / 6$ & $6 / 6$ & $6 / 6$ & $1 / 6$ & $6 / 6$ \\
\hline & 10 & $3 / 5$ & $3 / 5$ & $5 / 5$ & $0 / 5$ & $4 / 5$ \\
\hline & 6 & $1 / 4$ & $3 / 4$ & $2 / 4$ & $0 / 4$ & $2 / 4$ \\
\hline \multirow{5}{*}{$\begin{array}{l}\text { Foil/ } \\
\text { water }\end{array}$} & 50 & $5 / 5$ & $5 / 5$ & $5 / 5$ & $5 / 5$ & $5 / 5$ \\
\hline & 38 & $5 / 5$ & $5 / 5$ & $5 / 5$ & $1 / 5$ & $0 / 5$ \\
\hline & 15 & $3 / 5$ & $4 / 5$ & $5 / 5$ & $0 / 5$ & $2 / 5$ \\
\hline & 10 & $0 / 6$ & $5 / 6$ & $4 / 6$ & $0 / 6$ & $2 / 6$ \\
\hline & 6 & $3 / 4$ & $4 / 4$ & $2 / 4$ & $0 / 4$ & $1 / 4$ \\
\hline \multirow{5}{*}{$\begin{array}{l}\text { Foil/ } \\
\text { air }\end{array}$} & 50 & $5 / 5$ & $5 / 5$ & $5 / 5$ & $5 / 5$ & $5 / 5$ \\
\hline & 38 & $5 / 5$ & $5 / 5$ & $5 / 5$ & $5 / 5$ & $2 / 5$ \\
\hline & 15 & $2 / 8$ & $7 / 8$ & $8 / 8$ & $0 / 8$ & $1 / 8$ \\
\hline & 10 & $4 / 5$ & $4 / 5$ & $5 / 5$ & $0 / 5$ & $5 / 5$ \\
\hline & 6 & $4 / 4$ & $4 / 4$ & $4 / 4$ & $0 / 4$ & $0 / 4$ \\
\hline
\end{tabular}
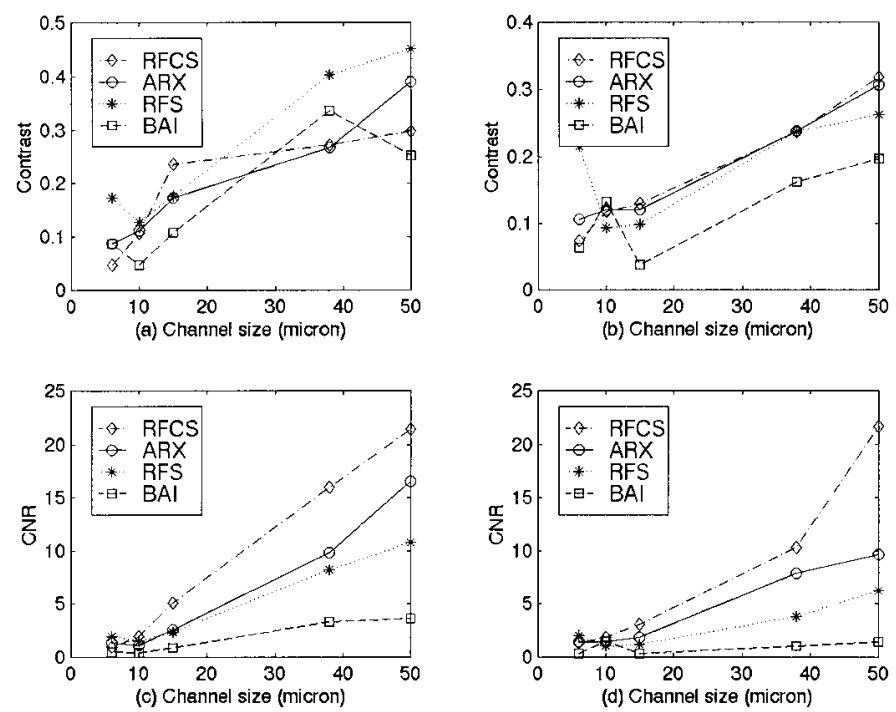

Fig. 7. a and c) Contrast and CNR for air-filled channels in plastic trilaminate film; b and d) contrast and CNR for water-filled channels in plastic trilaminate film. and high orders of parameters work well for small channels, i.e., $15 \mu \mathrm{m}$ or smaller. In future work parameter optimization should be investigated. Furthermore, in this work only the ARX model is being investigated because of its simple structure. Other more general models, such as ARMAX, ARARX, and ARARMAX, should be investigated to understand the performance of the parametric models completely. It could be expected that with the use of more complex model structure, the computational complexity would increase greatly.

\section{REFERENCES}

[1] K. L. Yam, "Pressure differential techniques for package integrity inspection," in Plastic Package Integrity Testing Assuring Seal Quality, B. A. Blakistone and C. L. Harper, Eds. Herndon, VA: Institute of Packaging Professional, 1995, ch. 17, pp. 137-145.

[2] R. A. Lampi, G. L. Schulz, T. Ciavarani, and P. T. Burke, "Performance and integrity of retort pouch seals," Food Technol., vol. 30, no. 2, pp. 38-48, Feb. 1976.

[3] K. Raum, A. Ozguler, S. A. Morris, and W. D. O'Brien, Jr., "Channel defect detection in food packages using integrated backscatter ultrasound imaging," IEEE Trans. Ultrason., Ferroelect., Freq. Contr., vol. 45, no. 1, pp. 30-40, Jan. 1998.

[4] C. H. Frazier, A. Ozguler, S. A. Morris, and W. D. O'Brien, Jr., "High contrast images of defects in food package seals," in Proc. 1997 IEEE Int. Ultrason. Symp., Toronto, ON, Canada, pp. 689-692.

[5] C. H. Frazier, Q. Tian, A. Ozguler, S. A. Morris, and W. D. O'Brien, Jr., "High contrast ultrasound images of defects in food package seals," IEEE Trans. Ultrason., Ferroelect., Freq. Contr., vol. 47, no. 3, pp. 530-539, May 2000.

[6] Q. Tian, A. Ozguler, S. A. Morris, and W. D. O'Brien, Jr., "High-contrast RF correlation imaging of defects in food package seals," presented at 1999 IEEE Int. Ultrason. Symp., Lake Tahoe, Nevada, 1999.

[7] L. Ljung, System Identification-Theory for the User. Englewood Cliffs, NJ: Prentice Hall, 1987.

[8] T. Söderström and P. Stoica, System Identification. London: Prentice Hall Int., 1989.

[9] J. Oppenheim and A. S. Willsky, Signals and Systems. Englewood Cliffs, NJ: Prentice Hall, 1985.

[10] S. W. Smith, R. F. Wagner, J. M. Sandrik, and H. Lopez, "Low contrast detectability and contrast/details analysis in medical ultrasound," IEEE Trans. Sonics Ultrason., vol. 30, no. 3, pp. 164-173, May 1983.

[11] D. H. Turnball, P. K. Lum, A. T. Kerr, and F. S. Foster, "Simulation of B-scan images from two-dimensional transducer arrays: Part I - Methods and quantitative contrast measurements," Ultrason. Imaging, vol. 14, pp. 323-343, 1992.

[12] S.H.P. Bly, D. Lee-Chahal, D. R. Foster, M. S. Patterson, F. S. Foster, and J. W. Hunt, "Quantitative contrast measurements in B-mode images comparison between experiment and theory," $U l$ trasound Med. Biol., vol. 12, no. 3, pp. 197-208, 1986.

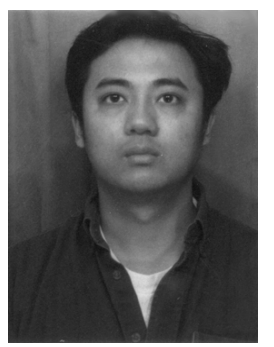

Qi Tian (S'95-M'96) was born in Chengdu, China on January 19, 1970. He received the B.E. degree in Electronic Engineering from Tsinghua University, P. R. China, in July 1992 and M.S. degree in Electrical Engineering from Drexel University, Philadelphia, PA, in June 1996. He is currently pursuing the Ph.D. degree in Electrical and Computer Engineering at the University of Illinois at UrbanaChampaign. He was a graduate research and teaching assistant in the Department of Electrical and Computer Engineering at Drexel University from 1994 to 1997 and a graduate research assistant in 
the Bioaccoustics Research Laboratory of the Beckman Institute for Advanced Science and Technology at UIUC from August 1997 to January 1999. Since February 1999, he has been a graduate research assistant at the Image Formation and Processing Group of the Beckman Institute for Advanced Science and Technology at UIUC.

His current research interests include multimedia content analysis and retrieval, digital image and signal processing, computer vision, and ultrasonic imaging. He has published over 15 journal and conference papers.

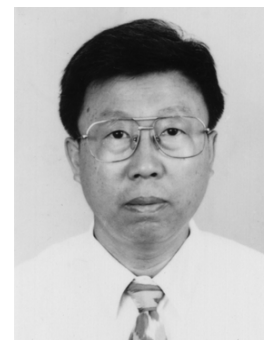

Bao-Shen Sun was born in TianJing, China on January 15, 1944. He graduated from the University of Science and Technology of China in 1967 in Electronics \& Acoustics and after that worked as an electronics engineer in a radio \& electronics factory. He attended the Graduate School, University of Science and Technology of China, from 1978 to 1981 to pursue a master's degree in Physics. From 1982 to 1985 , he was a visiting member of the Department of Engineering Science, Oxford University, UK, working on the study of the reflectivity and velocity perturbation caused by thin metal film structures on lithium niobate substrate. Since 1985, he has been at the Institute of Acoustic, Academia Sinica, where he is Research Professor of Acoustics. Currently, he is a visiting member in the Bioacoustics Research Laboratory, University of Illinois at UrbanaChampaign, and is going back to the Institute of Acoustics in Beijing soon. His research interests involve the SAW devices and their applications in signal processing, acoustic imaging, and synthetic aperture acoustic imaging.

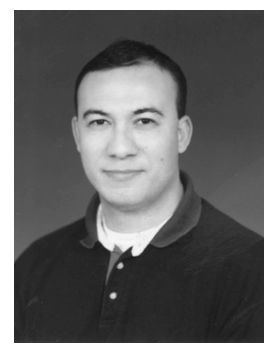

Ayhan Ozguler received B.Sc. degree in Food Engineering from Middle East Technical University, Ankara, Turkey, in 1991 and M.S. and Ph.D. degrees in Food Processing and Engineering from the University of Illinois, Urbana-Champaign, in 1995 and 1999, respectively.

From 1995 to 1999 , he was a research assistant at the University of Illinois and was involved in a joint research project between the Packaging Laboratory and the Beckman Bioacoustic Research Laboratory. His main research interests include nondestructive inspection techniques of food packages for the seal integrity and the ultrasonic imaging. Dr. Ozguler is currently employed as a postdoctoral research associate, and his responsibilities include quantifying the engineering trade-offs of using nondestructive ultrasonic sensor and defining the design parameters necessary for the safe, high speed production of shelf-stable foods in new energy- and material-efficient packages.

In 1993, he was awarded the scholarship of Republic of Turkey, Ministry of National Education to pursue master and Ph.D. degrees in the area of food packaging in the U.S. In 1998, he received the reward from the Packaging Education Forum for his contribution to the field of packaging. Dr. Ozguler is the member of Institute of Food Technologists (IFT) Food Packaging Division and Gamma Sigma Delta.

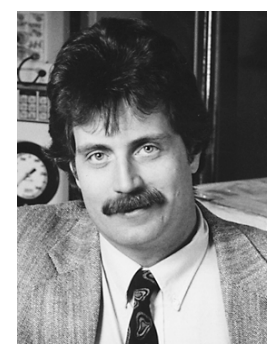

Scott A. Morris received the B.S., M.S., and Ph.D. degrees in 1981, 1987, and 1992 from Michigan State University.

While employed at Michigan State University, he worked with a variety of problems related to the packaging industry. Development of an experimental permeation modeling technique utilizing a combination of numerical modelling, optical measurement techniques, and laboratory analytical testing methods. Microwave cooking of frozen and shelf-stable foods, and the redesign of their related package systems. Improved shipping designs for retortable-pouch packaging systems. Development of chromatographic methods for the evaluation of volatile extractables from packaging materials. Determination of oxygen-barrier requirements of products that had been previously packaged in impermeable cans, and the evaluation of the suitability of producer-supplied polymeric replacements. Development of a research program in conjuction with a major packaging corporation, to test the ability of their 2-liter aseptic package to survive shipping and distribution. A survey of the state-of-the-art of packaging in the United States, and significant trends in the technical and economic aspects of the packaging industry commissioned by the Congressional Office of Technical Assessment (OTA). Investigating the relationship of package design to the effects of shock, vibration and compression on fresh and controlled-atmosphere-stored produces gaseous metabolic by-products.

Now an Associate Professor, Dr. Morris has been at the University of Illinois Departments of Food Science and Human Nutrition and Agricultural Engineering since 1992. He currently is developing a packaging research and teaching program. Current research projects within the program include: leak detection in shelf-stable food packages using high-frequency ultrasound, predictive modeling of package-structure permeation properties; static and dynamic structural modeling for design strength, and material-use optimization; packaging line efficiency optimization; product quality degradation from mechanical input and thermal variation; time-dependent material properties from stress-strain histories using recursive finite element algorithms; and microwave energy dispersion in food packages.

Dr. Morris is a member of the executive committee of the Institute of Food Technologists (IFT) Food Packaging Division, a member of the American Society of Agricultural Engineers (ASAE), Society of Professional Engineers (SPE), Institute of Packaging Professionals (IoPP), American Society for Engineering Education (ASEE), Pi Kappa Gamma, and Gamma Sigma Delta.

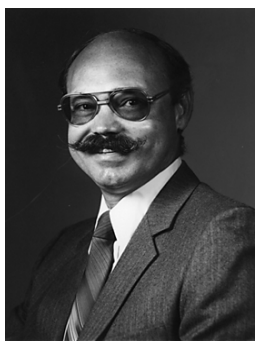

William D. O'Brien, Jr. (S'64-M'70SM'79-F'89) received the B.S., M.S., and Ph.D. degrees in 1966, 1968, 1970, from the University of Illinois, Urbana-Champaign.

From 1971 to 1975 he worked with the Bureau of Radiological Health (currently the Center for Devices and Radiological Health) of the U.S. Food and Drug Administration. Since 1975, he has been at the University of Illinois, where he is a Professor of Electrical and Computer Engineering, and Professor of Bioengineering, College of Engineering and of Bioengineering, College of Medicine. He is the Director of the Bioacoustics Research Laboratory and the Program Director of the NIH Radiation Biophysics and Bioengineering in Oncology Training Program. His research interests involve the many areas of acoustics and ultrasound, including spectroscopy, risk-assessment, biological effects, tissue characterization, dosimetry, blood-flow measurements, acoustic microscopy, and imaging. He has published more than 180 papers.

Dr. O'Brien is Editor-in-Chief of the IEEE Transactions on Ultrasonics, Ferroelectrics, and Frequency Control. He is a Fellow of the Institute of Electrical and Electronics Engineers (IEEE), the Acoustical Society of American (ASA), and the American Institute of Ultrasound in Medicine (AIUM), and he is a Founding Fellow of American Institute of Medical and Biological Engineering. He was recipient of the IEEE Centennial Medal (1984), the AIUM Presidential Recognition Awards (1985 and 1992), the AIUM/WFUMB Pioneer Awards (1988), the IEEE Outstanding Student Branch Counselor Award (1989), and the AIUM Joseph H. Holmes Basic Science Pioneer Awards (1993). He has been President (1982-1983) of the IEEE Sonics and Ultrasonics Group (currently the IEEE UFFC Society), Co-Chairman of the 1981 IEEE Ultrasonic Symposium, President of the AIUM (1988-1991), and Treasurer of the World Federation for Ultrasound in Medicine and Biology (1991-1994). He is the 19971998 IEEE UFFC Society's Distinguished Lecturer. 